\title{
Pseudoachondroplasia is caused through both intra- and extracellular pathogenic pathways
}

\author{
Robert Dinser, ${ }^{1}$ Frank Zaucke, ${ }^{1}$ Florian Kreppel, ${ }^{2}$ Kjell Hultenby, ${ }^{3}$ Stefan Kochanek, ${ }^{2}$ \\ Mats Paulsson, ${ }^{1}$ and Patrik Maurer ${ }^{1}$
}

${ }^{1}$ Institute for Biochemistry II, and

${ }^{2}$ Center for Molecular Medicine, Medical Faculty, University of Cologne, Cologne, Germany

${ }^{3}$ Clinical Research Center, Karolinska Institutet, Huddinge University Hospital, Stockholm, Sweden

\begin{abstract}
Pseudoachondroplasia is a dominantly inherited chondrodysplasia associated with mutations in cartilage oligomeric matrix protein (COMP). Investigations into the pathogenesis of pseudoachondroplasia are hampered by its rarity. We developed a cell culture model by expressing mutant COMP in bovine primary chondrocytes using a gutless adenoviral vector. We show that mutant COMP exerts its deleterious effects through both intra- and extracellular pathogenic pathways. Overexpression of mutant COMP led to a dose-dependent decrease in cellular viability. The secretion of mutant COMP was markedly delayed, presumably due to a prolonged association with chaperones in the endoplasmic reticulum (ER). The ECM lacked organized collagen fibers and showed amorphous aggregates formed by mutant COMP. Thus, pseudoachondroplasia appears to be an ER storage disease, most likely caused by improper folding of mutant COMP. The growth failure of affected patients may be explained by an increased cell death of growth-plate chondrocytes. Dominant interference of the mutant protein on collagen fiber assembly could contribute to the observed failure of the ECM of cartilage and tendons.
\end{abstract}

J. Clin. Invest. 110:505-513 (2002). doi:10.1172/JCI200214386.

\section{Introduction}

Pseudoachondroplasia (PSACH) is a dominantly inherited skeletal disorder caused by mutations in cartilage oligomeric matrix protein (COMP) $(1,2)$. It is characterized clinically by disproportionate dwarfism, a waddling gait, joint laxity, and severe premature osteoarthritis of weight-bearing joints (3). Some mutations in COMP can lead to a milder form of the disease, multiple epiphyseal dysplasia (MED) $(1,4)$. MED has also been associated with mutations in the genes coding for the $\alpha 1, \alpha 2$, and $\alpha 3$ chains of collagen IX and matrilin-3 (5-8). Electron microscopy of biopsies from affected patients showed that COMP, collagen IX, and aggrecan are retained in typical granular or lamellar inclusions of the endoplasmic reticulum (ER) of articular and growth-plate chondrocytes $(4,9-11)$ and of tenocytes $(12,13)$.

COMP is a secreted calcium-binding pentameric glycoprotein of the thrombospondin family, which is abundantly expressed in cartilage and to a lesser extent in tendons and ligaments (14-16). It consists of an

Received for publication October 10, 2001, and accepted in revised form June 29, 2002.

Address correspondence to: Robert Dinser, Medizinische Klinik I, Klinikum der Universität des Saarlandes, D-66421 Homburg, Germany. Phone: 49-6841-1623069; Fax: 49-68411623088; E-mail: robert.dinser@uniklinik-saarland.de. Conflict of interest: No conflict of interest has been declared. Nonstandard abbreviations used: Pseudoachondroplasia (PSACH); cartilage oligomeric matrix protein (COMP); tris buffered saline (TBS); multiple epiphyseal dysplasia (MED); endoplasmic reticulum (ER); cytomegalovirus (CMV); lactate dehydrogenase (LDH).
$\mathrm{N}$-terminal coiled-coil domain, followed by four EGFlike domains, eight calcium-binding thrombospondin type 3 repeats, and a C-terminal globular domain (17). In solid-phase assays, COMP has been shown to bind to collagens I, II, and IX in a zinc-dependent manner with a $K_{\mathrm{d}}$ of 3-30 $\mathrm{nM}(18,19)$. Despite its use as a marker for joint damage after trauma and during arthritis (20-22), its physiological role is largely unknown. A COMP knockout mouse has no apparent phenotype (23). The pathogenic pathway linking mutations of COMP to PSACH or MED is unclear.

Several hypotheses have been formulated. The intracellular accumulation of mutant COMP in chondrocytes could lead to a lack of COMP in the ECM. While some studies reported reduced amounts of COMP in the ECM of patient cartilage $(10,24)$, others showed that both patient and control chondrocytes could secrete COMP in cell culture $(11,25)$. Since only one allele is affected, both wild-type and mutant COMP will be expressed. Because no tools to distinguish mutant from wild-type COMP were available, it is not known whether mutant COMP can be secreted or whether only the wild-type COMP is processed and transported through the ER. An observational study suggested that chondrocytes with large inclusions undergo cellular necrosis (24). The reduced growth and the cartilage matrix instability could also be due to a lack of chondrocyte function.

If mutant COMP is secreted, a functional alteration may cause a weakened anchorage in the ECM. An altered binding to collagens I, II, and IX of COMP carrying PSACH-associated (D469 $\Delta$ ) and MED-associated 
(D361Y) mutations in the type 3 repeats has been observed in solid-phase assays (19), even though the collagen-binding site was localized to the C-terminal domain $(18,19,26)$. The calcium affinity of COMP was decreased, accompanied by conformational changes $(19,27)$. Similar results have been obtained with another PSACH-associated mutation, D446N (28). All published mutations leading to PSACH or MED are within the calcium-binding type 3 repeats or in the collagenbinding C-terminal domain. The potential importance of the COMP-collagen IX interaction is highlighted by the association of mutations in collagen IX genes with $\operatorname{MED}(5,6,8)$. Disease-associated mutant forms of COMP may disrupt the proper formation and organization of collagen fibers in patient tissues by dominant interference (19).

Further studies of the pathogenic mechanisms were hampered by the rarity of the disease and the difficulty in obtaining cartilage biopsies. Investigations of patient biopsies do not allow a differentiation between primary matrix alterations and secondary degenerative changes and remain descriptive in character. On the basis of previous work, where we optimized conditions for gene transfer and determined culture conditions allowing the retention of the chondrocyte phenotype $(29,30)$, we established a cell culture model, where the D469A COMP mutation is expressed through gene transfer in normal primary articular chondrocytes. We used highcapacity adenoviral vectors to achieve precise levels of transgene expression without toxic side effects $(29,31)$.

\section{Methods}

Materials. Low-viscosity alginate (Keltone LV) was obtained from C.P. Kelco (Chicago, Illinois, USA); ${ }^{35} \mathrm{~S}$-methionine EasyTag labeling mix and ${ }^{14} \mathrm{C}$-proline from NEN Life Science Products Inc. (Boston, Massachusetts, USA); rabbit anti-Grp78 and anti-calreticulin Ab's from StressGen Biotechnologies Corp. (Victoria, British Columbia, Canada); the PCEP4 plasmid from Invitrogen BV (Groningen, the Netherlands); ${ }^{3} \mathrm{H}$-proline from Amersham International (Buckinghamshire, United Kingdom); culture media and FBS from Gibco (Eggenstein, Germany); the mouse monoclonal (9E10) anti-c-myc Ab from Santa Cruz Biotechnology (Heidelberg, Germany); FuGene, protein A agarose, $N$-glycosidase $\mathrm{F}$, and endoglycosidase $\mathrm{H}$ from Roche Molecular Biochemicals (Mannheim, Germany); the monoclonal mouse B3-1 Ab against collagen IX from the Developmental Studies Hybridoma Bank (Iowa City, Iowa, USA). All other reagents came from SigmaAldrich Chemie GmbH (Deisenhofen, Germany). The $\mathrm{Ab}$ against COMP has been described previously (19). Cell culture. Primary articular chondrocytes were isolated by enzymatic digestion from bovine shoulder joints of 3- to 5-year-old animals obtained from the local slaughterhouse (32). In short-term studies, chondrocytes were cultured in monolayer for 5 days before gene transfer. Cultures were analyzed 3 days later. For long-term experiments, the cells were transduced immediately after isolation and subsequently embedded in alginate (32). Cultures were analyzed after 2-5 weeks. The culture medium consisting of DMEM/F12 supplemented with $10 \%$ FCS and $100 \mu \mathrm{M}$ ascorbate was changed twice weekly.

Vectors and gene transfer. We used a gutless adenoviral vector as described previously (29). The expression cassette consisted of the cytomegalovirus (CMV) immediate early promoter controlling the expression of a fusion protein of the BM- 40 signal peptide, an $\mathrm{N}$-terminal His $c$-myc factor X tag (33), and full-length rat COMP (19), followed by the SV40 polyadenylation signal. Two constructs, one with the wild-type COMP sequence and one with a sequence carrying the most frequent PSACHassociated mutation, D469 , were used (19). The plasmid pFK1 used to construct the adenoviral vectors was generated by inserting the blunt-ended SalI/SalI fragment from the pCEP4 plasmid into the blunt-ended NotI site of the shuttle plasmid pSTK129 (29). The cDNA of the fusion protein was cloned into the plasmid, and vectors were amplified as described previously (29). The infectious titers of the adenoviral vectors were determined by a slot blot procedure on HeLa cells in duplicate experiments (34). The titers were $1 \times 10^{7}$ and $2 \times 10^{7}$ infectious units per microliter for the vector coding for mutant and wild-type COMP.

For transductions of monolayer cultures, a defined moi of the adenoviral vector was added with $0.75 \mathrm{ml}$ of fresh culture medium to each well of a six-well plate containing 300,000 adherent cells and incubated at $37^{\circ} \mathrm{C}$. Cell numbers and plate size were scaled up as needed (29). For alginate cultures, the adenovirus was added to a suspension of $10^{7}$ freshly isolated chondrocytes in 2.5 $\mathrm{ml}$ of $1.2 \%$ alginate in $150 \mathrm{mM} \mathrm{NaCl}$. After 30 minutes at $37^{\circ} \mathrm{C}$, the alginate was allowed to gel in $100 \mathrm{mM} \mathrm{CaCl}_{2}$ (32). Except for the cell viability assays, all experiments were performed with a low (20 moi) or a high (100-200 moi) multiplicity of infection.

Cell viability assay. The viability of chondrocytes overexpressing COMP was assessed with a tetrazolium salt colorimetric assay (35) 3 days after adenoviral gene transfer into monolayer cells using increasing infectious titers. Following gene transfer, cells were cultured under serum-free conditions. Results were expressed as relative viable cell number compared with untransfected control chondrocytes.

Lactate dehydrogenase activity assay. Three days after transduction of monolayer cultures $(300,000$ cells per well of a six-well- plate), the cell culture supernatant was harvested, centrifuged, and the total lactate dehydrogenase (LDH) activity measured in the Central Laboratory of the Cologne University hospital using standard methods. Measured values were corrected for the background activity of the culture medium and related to the LDH activity of a total cell lysate obtained by sonication of 300,000 chondrocytes.

Metabolic labeling of COMP. The kinetics of COMP secretion were assessed in pulse-chase experiments. Cells were starved for 2 hours in cysteine- and 
methionine-free medium, pulsed for 1 hour with 100 $\mu \mathrm{Ci} / \mathrm{ml}$ medium of ${ }^{35} \mathrm{~S}$-methionine, and chased in normal medium. After harvest of culture supernatants, monolayer cells were lysed in 1\% NP-40, 50 $\mathrm{mM}$ Tris, pH 7.4 (lysis buffer). Alginate beads were dissolved in $55 \mathrm{mM}$ sodium citrate, $150 \mathrm{mM} \mathrm{NaCl}$, pH 6.8 (solubilization buffer), and gently centrifuged (32). The citrate-soluble matrix in the supernatant was harvested and the pellet treated with lysis buffer. Samples were stored at $-20^{\circ} \mathrm{C}$. Pulse-chase experiments were carried out three days after gene transfer for short-term studies except where mentioned otherwise. Analysis of long-term alginate cultures was carried out 3 days after a 2-hour pulse with 100 $\mu \mathrm{Ci} / \mathrm{ml}$ medium of ${ }^{35} \mathrm{~S}$-methionine.

Immunoprecipitation of COMP. Ab's against COMP or $c$-myc (final dilution 1:150 and 1:75, respectively) were coupled to protein A agarose for 3 hours at room temperature. Unspecific binding was blocked with $10 \%$ BSA. Samples were precleared in parallel with protein $\mathrm{A}$ agarose. The protein $\mathrm{A}-\mathrm{Ab}$ complexes were added and the mixtures incubated overnight at $4^{\circ} \mathrm{C}$. After precipitation, the samples were washed three times with $0.5 \%$ Triton-X 100 in tris buffered saline (TBS). COMP was eluted by boiling the samples for 5 minutes in sample buffer containing 5\% mercaptoethanol except when nonreducing conditions were used explicitly. For nonreducing electrophoresis, the samples were heated to $85^{\circ} \mathrm{C}$ for 30 minutes in normal sample buffer. Electrophoresis was performed with $6 \%$ or $4 \%$ polyacrylamide gels. The gels were incubated in $1 \mathrm{M}$ sodium salicylate and dried before exposure of $\mathrm{x}$-ray films or quantification of labeled COMP with a PhosphoImager and the ImageQuant software (Molecular Probes, Krefeld, Germany).

Coimmunoprecipitation. Three days after transduction of monolayer cultures with 100 moi, immunoprecipitation was performed on the cell lysates using the antiGrp78 and anti-calreticulin Ab's (final dilution 1:125), with two modifications to the above protocol. The lysis buffer included $150 \mathrm{mM} \mathrm{NaCl}$, and the precipitated samples were washed with $0.05 \%$ Triton X-100 in TBS $(36,37)$. The polyacrylamide gels were subjected to immunoblotting with a primary $\mathrm{Ab}$ directed against $c$-myc (diluted 1:2,000).

Oligosaccharide analysis. The glycosylation pattern was analyzed 3 days after gene transfer with mutant COMP. Cells were pulsed with ${ }^{35} \mathrm{~S}$-methionine as above, chased for 4 hours, and the cell lysate and supernatant immunoprecipitated with Ab's against $c$-myc. The precipitates were directly digested with $\mathrm{N}$-glcyosidase F and endoglycosidase $\mathrm{H}$, respectively, following the manufacturers protocol, before analysis by SDS-PAGE and fluorography.

Metabolic labeling of collagens. Collagen II secretion was analyzed in monolayer culture three days after transduction with 100 moi. After a 2-hour incubation in serum-free medium, cells were pulsed for 1 hour in the presence of $100 \mu \mathrm{g} / \mathrm{ml} \beta$-aminopropionitrile and
$10 \mu \mathrm{Ci} / \mathrm{ml}$ of ${ }^{14} \mathrm{C}$-proline, followed by a chase in normal medium supplemented with $\beta$-aminopropionitrile (100 $\mu \mathrm{g} / \mathrm{ml})$ and fresh ascorbic acid $(100 \mu \mathrm{M})$. The supernatant was harvested, precipitated by the addition of ammonium sulphate to $30 \%$ saturation, and digested with $100 \mu \mathrm{g} / \mathrm{ml}$ pepsin in $0.5 \mathrm{M}$ acetic acid at $4^{\circ} \mathrm{C}$ for 3 days. Samples were dialyzed against $100 \mathrm{mM}$ ammonium bicarbonate and lyophilized before SDS-PAGE under reducing conditions.

For steady-state collagen analysis after 5 weeks of alginate culture following transduction with 200 moi, the culture medium was supplemented with $100 \mu \mathrm{M}$ of fresh ascorbic acid and with $10 \mu \mathrm{Ci} / \mathrm{ml}$ of ${ }^{3} \mathrm{H}$-proline. The next 4 days the supernatant was harvested and fresh labeling medium added. Three days later, the supernatant was again harvested and the alginate beads dissolved in solubilization buffer. The supernatants and the citrate soluble matrix fraction were precipitated with ammonium sulphate. The pellets were treated as just described. An identical number of alginate beads was used for each experiment, so that no adjustment was made for changes in cell number during culture.

Immunohistochemistry of alginate beads. Chondrocytes were transduced with 100 moi and cultured for 5 weeks in alginate. Beads were embedded in paraffin, processed, and stained as described previously (30), using the undiluted cell culture supernatant of the $\mathrm{Ab}$ against collagen IX.

Electron microscopy. For electron microscopy, alginate beads were fixed in $2 \%$ glutaraldehyde in $0.1 \mathrm{M}$ sodium cacodylate, pH 7.3, containing $5 \mathrm{mM} \mathrm{CaCl}_{2}$. Samples were rinsed in $0.15 \mathrm{M}$ sodium cacodylate, $\mathrm{pH} 7.3$, postfixed in $1 \%$ osmium tetroxide in $0.1 \mathrm{M}$ sodium cacodylate, dehydrated in ethanol followed by acetone, and embedded in LX-112. Sections were contrasted by uranyl acetate followed by lead citrate and examined in a Tecnai 10 transmission electron microscope at $80 \mathrm{kV}$. For immunogold labeling, alginate beads were fixed in $3 \%$ paraformaldehyde, $0.1 \%$ glutaraldehyde in $0.1 \mathrm{M}$ sodium cacodylate buffer, $\mathrm{pH} 7.3$, containing $5 \mathrm{mM}$ $\mathrm{CaCl}_{2}$. Samples were immersed in $2.3 \mathrm{M}$ sucrose, frozen in liquid nitrogen and cryosectioned at $-100^{\circ} \mathrm{C}$. Labeling was performed as described previously, using the anti-COMP Ab in a 1:200 dilution, detecting with 10 nm gold-conjugated protein A (38).

\section{Results}

Experimental system. We expressed a mutant form of COMP with a deletion of Asp in position 469 (D4694) causing PSACH (19) and a wild-type form in primary articular chondrocytes. Both forms were fused with a $c$-myc tag at the $\mathrm{N}$-terminus of the pentamerization domain to distinguish them from the endogenous COMP produced by articular chondrocytes. Gene transfer was achieved by transduction with a gutless third-generation adenoviral $(29,31)$.

The conditions for culture were chosen such that dedifferentiation of chondrocytes was negligible. Shortterm studies were completed within 8 days after isolation. 

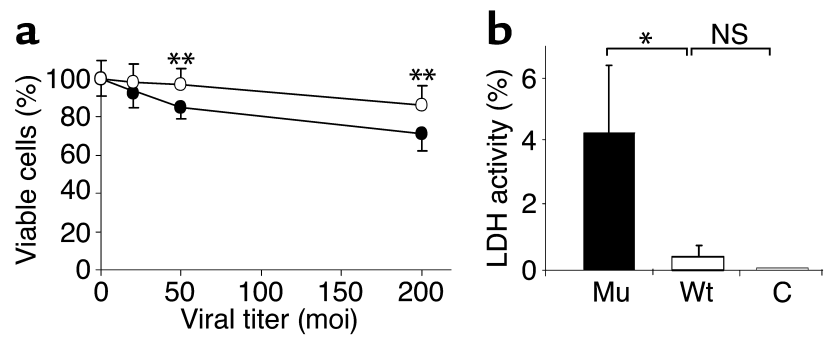

Figure 1

Overexpression of mutant COMP decreases the viability of chondrocytes. (a) Monolayer cells were transduced with different infectious titers of adenovirus, and viable cells were quantified 3 days later. Viable cells are indicated relative to untransduced control cultures. Filled circles, cultures expressing mutant COMP; open circles, cultures expressing wild-type COMP. The composite result of two independent experiments is shown, with each point representing nine measurements. Error bars indicate $1 \mathrm{SD} ;{ }^{*} P<0.01$ in the two-sided student $t$ test. (b) The supernatants of cultures were analyzed for LDH activity 3 days after gene transfer with 200 moi. Values were corrected for the background activity of the culture medium and related to the LDH activity of a total cell lysate obtained by sonication of an equivalent cell number. Measurements were performed in triplicate, error bars indicate 1 SD. NS, no significant difference; ${ }^{*} P<0.05$ in the two-sided student $t$ test. Mu, cultures overexpressing mutant COMP; $\mathrm{Wt}$, cultures overexpressing wildtype COMP; $\mathrm{C}$, untransduced control cultures.

Long-term studies were performed in alginate culture where the chondrocytic phenotype is largely maintained $(30,32)$. Previous experiments with the same vector expressing enhanced green fluorescent protein showed that the transfection efficiency was $82 \%$ with 100 moi of the adenoviral vector. After 6 weeks of culture, $64 \%$ of the adenovirally transduced chondrocytes remained transgene positive (29).

Expression of a PSACH-associated COMP mutant affects cellular viability. The effect of exogenous COMP on cellular viability was assessed in monolayer culture by transducing chondrocytes with increasing infectious titers of the adenoviral vector. The number of viable cells decreased in a dose-dependent manner with increasing expression of mutant COMP. Overexpression of wild-type COMP led to a less-pronounced effect on cellular viability (Figure 1a), which may be explained through nonspecific effects of the vector or the exogenous protein. The activity of the intracellular enzyme lactate dehydrogenase was increased in the supernatant of mutant COMP-expressing cultures, showing a disruption of the plasma membrane and indicating cell death (Figure 1b). Subsequent studies were performed with a low viral dose (20 moi), where only weak impairment of viability was observed, and a high viral dose (100-200 moi) to maximize toxic effects of mutant COMP.

Mutant COMP is secreted from chondrocytes at a delayed rate. Immunoprecipitation using an $\mathrm{Ab}$ against $c$-myc allowed us to demonstrate that mutant COMP is secreted at a delayed rate from chondrocytes in monolayer culture (Figure 2a). We did not observe an increased presence of COMP degradation products in the cell lysates (Figure 2a), and mutant COMP was assembled and secreted as a pentamer (Figure 2b), in agreement with previous studies of other COMP mutations $(10,25)$. Immunoprecipitation with an antiserum against COMP showed that differentiated chondrocytes secrete high amounts of recombinant wild-type COMP at the same rate as endogenous COMP. In contrast, secretion of mutant COMP was delayed, and significant amounts were still found in the cell lysate after a chase of 96 hours (Figure 2c).

The distribution of COMP within alginate cultures was studied 5 weeks after gene transfer (Figure 2, $d$ and e). More mutant than wild-type or endogenous COMP remained in the cellular fraction both after gene transfer with low (Figure 2d) and high (Figure 2e) infectious titers. Most endogenous, wild-type, or mutant COMP was recovered in the culture supernatant, indicating a weak anchorage within the matrix formed by the chondrocytes in alginate. Using low infectious titers, similar amounts of mutant, wild-type, and endogenous COMP were recovered in the citrate-soluble matrix, suggesting that the overall retention of COMP in the matrix formed in alginate was not severely altered by the mutation (Figure 2d). The sum of the COMP activity detected in the supernatant, citrate, and cell lysate fraction following gene transfer with 20 moi of the vector coding for mutant or wild-type COMP was twice as high as in control cultures (Figure 2d), indicating that amounts of exo- and endogenous COMP were equivalent. When using a tenfold infectious titer, the COMP activity recovered increased proportionally after transfer of the gene coding for wild-type COMP, whereas the amount of COMP in mutant COMP-expressing cultures was only three- to fourfold higher than in untransduced control cultures (Figure 2e), providing additional evidence for the toxicity of mutant COMP.

The secretory delay occurs in the ER and not in the Golgi compartment. Electron microscopy of chondrocytes cultured in alginate after transduction with a low infectious titer of the virus coding for mutant COMP showed an extensive distention of the rough ER (Figure 3, a and b), whereas high-level overexpression of wild-type COMP led only to slight alterations of cellular morphology (Figure 3c). High overexpression of mutant COMP led to the formation of granular inclusions in the ER (Figure 3, d and e). COMP was detected within these inclusions by immunogold labeling (Figure 3f). The morphological differences observed between cells in cartilage biopsies and those cultured in alginate are most likely due to different levels of expression of mutant COMP and differing nutritive milieus and mechanical stresses.

The retention of mutant COMP in the ER, and not in the Golgi compartment, was confirmed by the analysis of the glycosylation pattern of intracellular COMP. Intracellular mutant COMP was sensitive to endoglycosidase $\mathrm{H}$ digestion, indicating a high-mannose glycosylation consistent with a localization of COMP within the ER (Figure 3g). Extracellular mutant COMP was 
a

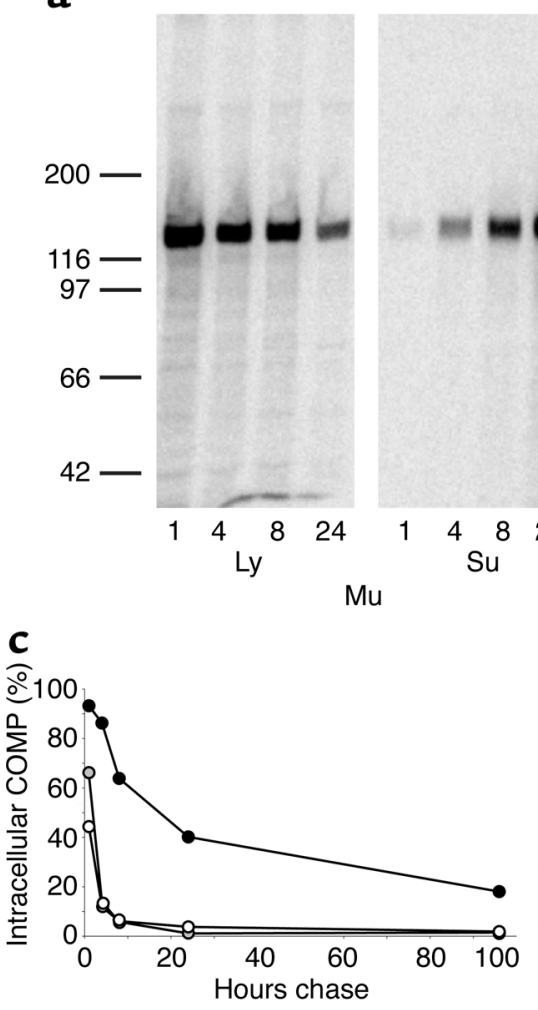

b
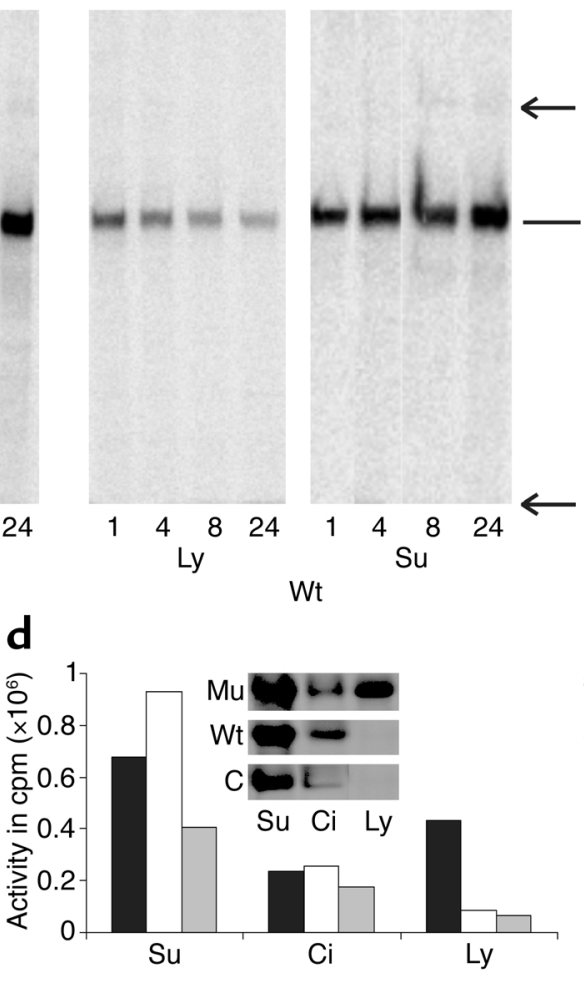
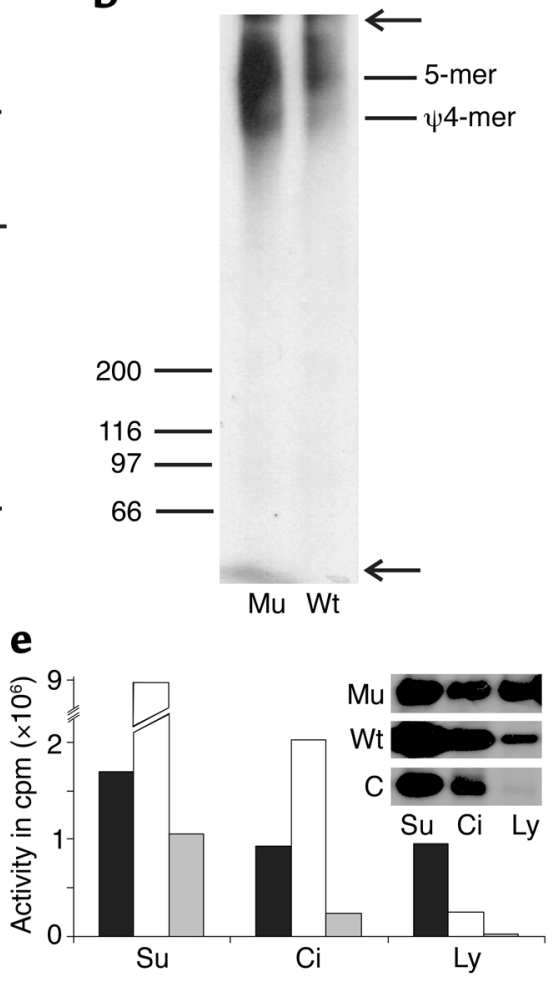

Figure 2

The secretion of mutant COMP is delayed but not abolished. (a) The secretion of wild-type (Wt) or mutant (Mu) COMP in monolayer chondrocytes transduced with 200 moi was analyzed after c-myc specific immunoprecipitation in pulse-chase experiments. Ly, cell lysates; Su, supernatants. The hours of chase are indicated below the gels. Molecular weight standards are shown on the left, the position of reduced recombinant COMP on the right. Arrows indicate origin and front of the separating gel. (b) Cell lysates of chondrocytes treated as above and chased for 30 minutes were subjected to gel electrophoresis under nonreducing conditions after $c$-myc specific immunoprecipitation. The position of recombinant COMP multimers is indicated on the right side of the gel. $\Psi 4$-mer indicates a pseudotetramer (19). (c) A pulse chase experiment of one-week-old alginate cultures transduced with 200 moi followed by COMP-specific immunoprecipitation shows the proportion of COMP found in the cell lysate versus total COMP. Closed symbols represent mutant COMP, open symbols wild-type COMP, shaded symbols endogenous COMP from untransduced chondrocytes. (d and e) Chondrocytes were transduced with 20 (d) or 200 (e) moi, cultured in alginate for five weeks, and pulse-labeled. The COMP-specific precipitated activity in supernatant (Su), citrate soluble alginate fraction $(\mathrm{Ci})$, and cell lysate $(\mathrm{Ly})$ is indicated. Black bars represent cultures expressing mutant COMP $(\mathrm{Mu})$, white bars represent wild-type $\operatorname{COMP}(\mathrm{Wt})$, and gray bars represent controls $(\mathrm{C})$. The insets show radiographs of the gels.

sensitive only to $\mathrm{N}$-glycosidase $\mathrm{F}$, an enzyme cleaving both high-mannose and complex-type glycans, showing that the transfer to the Golgi compartment, but not the processing there, is affected by the mutation (39).

Mutant and wild-type COMP were coprecipitated with Ab's to Grp78 and calreticulin (Figure $3 \mathrm{~h}$ ), two ER proteins that chaperone the correct folding of newly synthesized proteins (40). Both chaperones thus seem to interact with COMP. The stronger signal observed for mutant COMP presumably reflects a prolonged interaction of mutant compared with wild-type COMP with these chaperones or the increased availability of mutant COMP in the ER.

The structure of the ECM is severely altered. Electron microscopy revealed that the morphology of the ECM was severely disturbed by the expression of mutant COMP. Amorphous electron-dense aggregates were found throughout the pericellular compartment both after transduction with a low or high viral titer (Figure
4 , a and $b)$. Collagen fibrils remained unorganized and thin (Figure 4, a and b). The regular pattern of proteoglycan-derived nodules along the fibrils was abolished. Immunogold labeling showed that the amorphous aggregates contain COMP (Figure 4c). The matrix formed by chondrocytes overexpressing wild-type COMP was characterized by an abundant collagen deposition with occasional formation of thick collagen fibers (Figure 4d). These fibers appeared less organized than those formed by untransduced chondrocytes (Figure 4e). Chondrocytes overexpressing wild-type COMP also differed from untransduced cells by the appearance of additional electron-dense nodules next to the collagen fibrils.

This difference in matrix morphology was not due to a lack of collagen II or IX. Immunohistochemical staining of alginate beads showed that collagen IX was retained within mutant COMP-expressing chondrocytes, but also revealed a deposition of collagen IX in the pericellular 

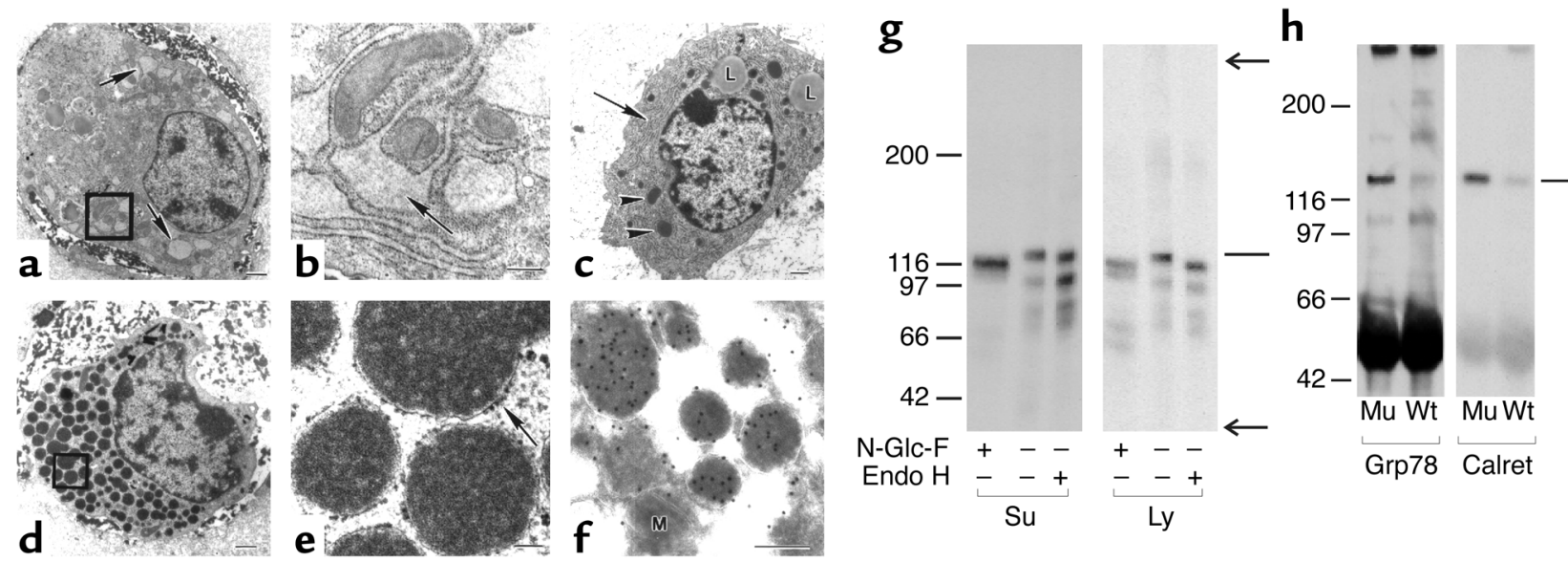

Figure 3

Mutant COMP is retained in the endoplasmic reticulum of chondrocytes. (a-f) Chondrocytes transduced with 20 moi (a and b) or 200 moi (c-f) were cultured in alginate for two weeks before ultrastructural analysis. Low-level expression of mutant COMP (a) leads to a marked distention of the ER (arrows). (b) Magnification of a. (c) A cell with a high-level overexpression of wild-type COMP exhibits a normal ER (arrow) with occasional formation of granular structures (arrowhead). L, lipid droplets. Chondrocytes overexpressing mutant COMP reveal multiple granular structures (d) that are surrounded by a membrane carrying ribosomes, thus corresponding to granular inclusions in the ER (arrow). (e) Magnification of d. Immunogold-labeling shows that the inclusions contain COMP (f). M, mitochondriae. Bars in a, $\mathbf{c}, \mathbf{d}$, $1 \mu \mathrm{m} ; \mathbf{b}$ and f, $200 \mathrm{~nm} ; \mathbf{e}, 100 \mathrm{~nm}$. (g) Cell lysates (Ly) and supernatants (Su) from transfected pulse-labeled monolayer cultures were digested with $\mathrm{N}$-glycosidase $\mathrm{F}(\mathrm{N}-\mathrm{Gl} \mathrm{c}-\mathrm{F})$, endoglycosidase $\mathrm{H}$ (EndoH), or left untreated after $\mathrm{c}$-myc specific precipitation. Molecular weight standards are indicated on the left side of the gels, recombinant COMP on the right side. The arrows mark origin and front of the separating gel. (h) Lysates of transduced monolayer cultures were precipitated with antibodies against Grp78 or calreticulin (Calret) and immunoblotted $\mathrm{Mu}$, mutant; $\mathrm{Wt}$, wild-type COMP expressing cultures.

matrix, suggesting that collagen IX was still secreted (Figure 5 , a-f). The secretion of collagen II was not affected by the overexpression of mutant COMP (Figure $5 \mathrm{~g}$ ), and the overall distribution of collagen II in alginate was similar in cultures overexpressing mutant or wild-type COMP and in untransduced control cultures (Figure $5 \mathrm{~h}$ ).

\section{Discussion}

Expression of the PSACH-associated D4694 mutation of COMP in cell culture resulted in a severe intra- and extracellular pathology, in keeping with different parallel pathogenic pathways of the disease.

\section{Figure 4}

The matrix formed by cultures expressing mutant COMP is disturbed. Chondrocytes were fixed after 2 weeks of alginate culture following adenoviral transduction with 20 moi (a) or 200 moi (b-d). Electron microscopy of the pericellular matrix of cultures expressing low levels of mutant COMP reveals electron-dense amorphous aggregates (arrow) and some sparse collagen fibrils (a, arrowhead). The matrix formed after transduction with 200 moi of the vector carrying the gene for mutant COMP has a similar structure, with amorphous aggregates (arrows) and thin collagen fibrils (arrowheads) lacking the regular proteoglycan stain (b). (c) Immunogold-labeling reveals a high concentration of COMP labeling in the amorphous aggregates compared with the low concentration next to the collagen fibers (arrowheads). (d) Chondrocytes overexpressing wild-type COMP form thick collagen fibers (arrow) carrying abundant electron-dense nodular structures (arrowheads). (e) The matrix formed by untransduced chondrocytes shows abundant thick collagen fibers (arrow) with occasional nodular structures corresponding to proteoglycans (arrowheads). Bars, $100 \mathrm{~nm}$.
We found that the expression of mutant COMP is toxic to previously healthy articular chondrocytes. The release of a cytosolic enzyme indicated a disruption of the plasma membrane induced by the overexpression of mutant COMP. A descriptive study using patient chondrocytes described necrotic cell death after several months of culture (24). This decrease in viability may be more relevant to growth zone cartilage than to articular

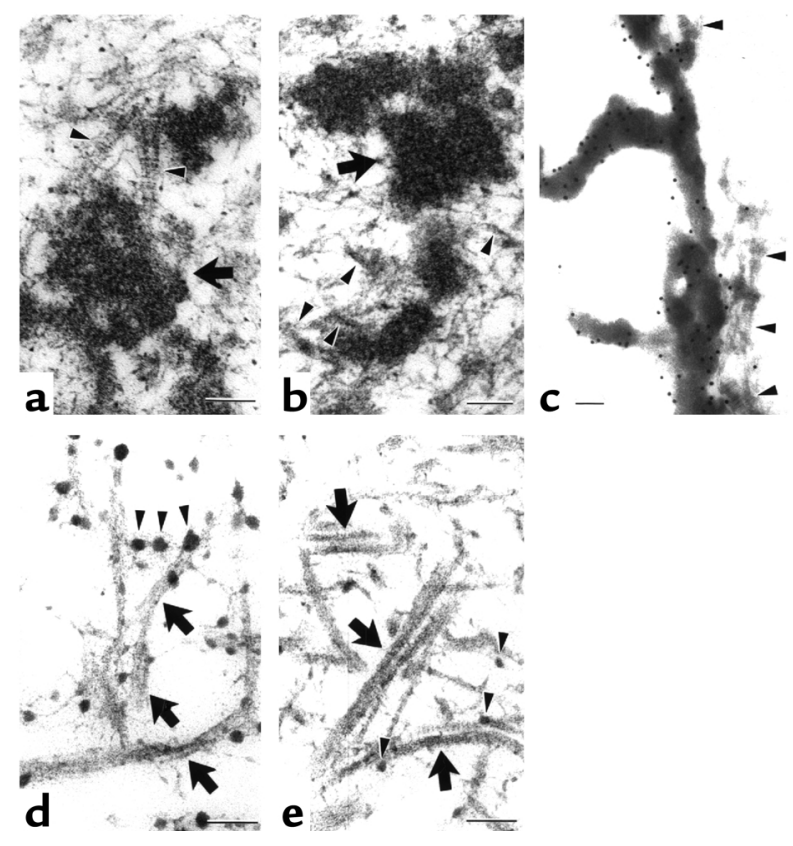


cartilage in-vivo, because COMP synthesis is considered to be higher in hypertrophic chondrocytes $(41,42)$ and could explain the short stature of affected patients.

The secretion of mutant COMP by fully differentiated chondrocytes was markedly delayed, but not completely abolished. Previous studies have been inconclusive in this regard. Secretion was shown with metabolic labeling for dedifferentiated patient chondrocytes only $(10,11,25)$, whereas differentiated cells have only been analyzed histologically $(10,24)$. We show that the secretory delay occurs within the ER, whereas the passage through the Golgi compartment is not affected by the mutation.

The intracellular persistence may be explained by misfolding or delayed folding of the mutant chains. We observed a strong binding of mutant COMP to two ER-based chaperones, Grp78 and calreticulin. Both have been implicated in the folding of oligomeric glycoproteins $(36,43)$. Conformational alterations of the D469 $\Delta$ and another PSACH-associated mutation have been reported from in vitro studies $(19,27,28)$. It is reasonable to assume that a prolonged binding of mutant COMP to chaperones is the cause for the retention in the ER. PSACH is thus an ER storage disease (39) caused by the improper folding of mutant COMP. Interestingly, even though COMP accumulated in the ER, no increase in degradation could be detected. The quality control system in the ER should recognize and degrade terminally unfolded molecules (38). Apparently, mutant COMP escapes this quality control. Whether this is due to an aggregation of COMP or to a complex formation in the ER with collagen IX and/or other factors is unclear.

In addition to these cellular phenotypes, we observed a misassembly of the ECM by chondrocytes expressing mutant COMP, even though the overall distribution of COMP and collagen II was not affected. Electron microscopy revealed amorphous electron dense aggregates containing COMP in the pericellular compartment of these cells. Organized
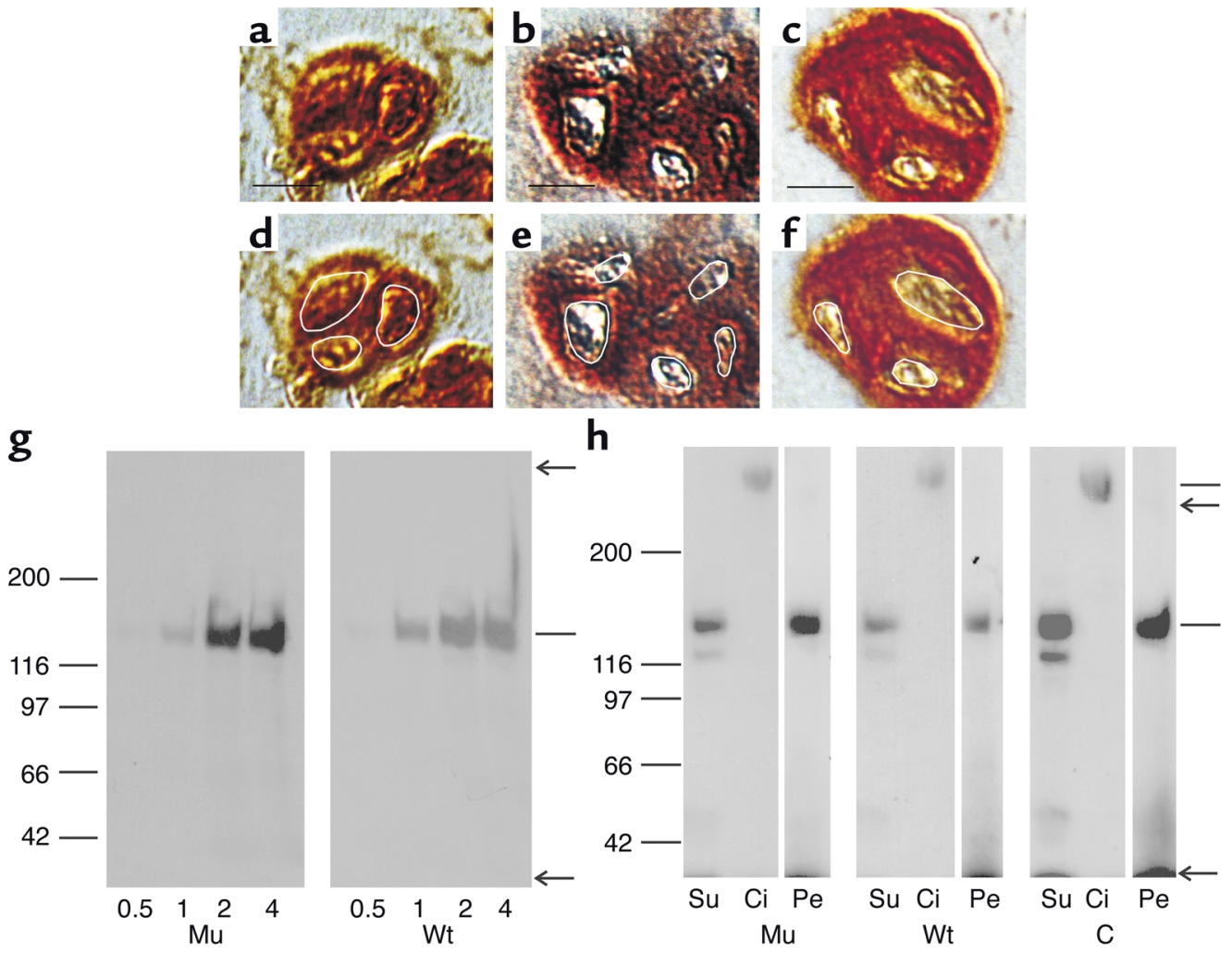

\section{Figure 5}

Collagen II and IX are deposited in the pericellular matrix. (a-f) Alginate beads were embedded in paraffin after five weeks of culture and sections stained with an antibody against collagen IX. Images $\mathbf{a}-\mathbf{c}$ are duplicates of images $\mathbf{d}-\mathbf{f}$, except that cell borders are outlined with a white line in the latter series. $\mathbf{a}$ and $\mathbf{d}$ show chondrocytes expressing mutant COMP, $\mathbf{b}$ and $\mathbf{e}$ cultures overexpressing wild-type COMP, $\mathbf{c}$ and f untransduced cultures. Bars indicate $10 \mu \mathrm{m}$. (g) Collagen II secretion was assessed by pulse-chase experiments in cells expressing mutant $(\mathrm{Mu})$ and wild-type $(\mathrm{Wt})$ COMP. Numbers below the gels indicate the hours of chase. The positions of the molecular weight standards are indicated on the left, of collagen II to the right. The arrows mark origin and front of the separating gel. (h) The distribution of collagen II in the culture supernatant $(\mathrm{Su})$, the citrate soluble alginate matrix ( $\mathrm{Ci})$, and the citrate resistant pellet (Pe) was evaluated after five weeks of alginate culture. The lanes showing the collagen II content of the citrate resistant pellets were exposed for a longer time than the other lanes. The position of cross-linked and pepsin-digested collagen II is indicated on the right side. The lower band observed exclusively in the supernatants represents the $\alpha 2$-chain of collagen I, produced at the outer surface of alginate beads $(30,32)$. Densitometric analysis revealed no significant variation in the proportion of collagen I to collagen II (32) between control and tranfected cultures. 
collagen fibers were lacking. While another study analyzing collagen fibril morphology did not observe a difference between patient and control chondrocytes after prolonged dedifferentiation in cell culture, our findings are similar to those in patient cartilage biopsies (24).

This disruption of the organization of collagen fibers could be due to a direct effect of mutant COMP on fiber assembly or to the intracellular retention of other components essential for the fiber formation like collagen IX. An altered binding of mutant COMP to collagen II and IX has been found in vitro using purified proteins (19). A lack of collagen II or IX seems unlikely. We observed an extracellular staining for collagen IX in an immunohistochemical analysis and the distribution of collagen II within the alginate gel was not influenced by the synthesis of mutant COMP. Overexpression of wild-type COMP also disturbed collagen fiber formation, albeit to a lesser degree. This could be due to an increased ratio of COMP to collagens and points to a role for COMP in regulating collagen fiber assembly (44), even if mice deficient in COMP do not show any apparent phenotype (23). The D469 $\Delta$ COMP mutation may cause the observed disruption of the organization of the ECM by dominantly interfering with collagen fiber assembly, providing another pathogenic element for PSACH.

Some difficulties of this cell culture model should be considered. We analyzed a disease within days to weeks of culture, which in humans takes months to years to develop. We took care to adapt the concentration of exogenous to that of endogenous COMP, but the time course of COMP production following viral infection is not equivalent to the regulation of endogenous COMP synthesis. Even though we did not observe any difference in oligomerization between the exogenous $c$-myc-tagged and the endogenous COMP, we cannot exclude subtle abnormalities that may contribute to the phenotype observed. Nevertheless, the clear differences observed after gene transfer of the mutant and the wild-type protein throughout the viral dose range used support the validity of our model.

In summary, our cell culture model of PSACH provides data to support parallel intra- and extracellular pathways to the pathogenesis of the disease: (a) PSACH is an ER-storage disease presumably caused by improper folding of mutant COMP and prolonged binding to ER chaperones; (b) the retention of mutant COMP in the ER leads to an increased cell death, explaining the growth failure of affected patients; (c) mutant COMP disrupts the formation of a proper ECM, providing an explanation for the ligamentous laxity and the early joint failure observed in PSACH individuals. The combination of gene transfer using an adenoviral vector devoid of viral proteins and negligible toxicity with long-term culture of primary chondrocytes in an alginate gel should allow insights into other dominantly inherited cartilage diseases.

\section{Acknowledgments}

We gratefully acknowledge the help of Christof Geisen from the Central Laboratory of the University of Cologne with the measurements of lactate dehydrogenase activity. This work was supported by grants from the Köln Fortune program of the Medical Faculty of the University of Cologne (R. Dinser), from the European Commission Biomed 2 program (contract number BMH4-CT97-2437, M. Paulsson), from the Boehringer Ingelheim Foundation (F. Kreppel), and from the Swedish Medical Research Council (K. Hultenby).

1. Briggs, M.D., et al. 1995. Pseudoachondroplasia and multiple epiphyseal dysplasia due to mutations in the cartilage oligomeric matrix protein gene. Nat. Genet. 10:330-336.

2. Hecht, J.T., et al. 1995. Mutations in exon 17B of cartilage oligomeric matrix protein (COMP) cause pseudoachondroplasia. Nat. Genet. 10:325-329.

3. International Working Group on Constitutional Diseases of Bone. 1998. International nomenclature and classification of osteochondrodysplasias. Am. J. Med. Genet. 79:376-382.

4. Stanescu, R., Stanescu, V., Mureil, M.P., and Maroteaux, P. 1993. Multiple epiphyseal dysplasia, Fairbank type: morphologic and biochemical study of cartilage. Am. J. Med. Genet. 45:501-507.

5. van Mourik, J.B., Hamel, B.C., and Mariman, E.C. 1998. A large family with multiple epiphyseal dysplasia linked to COL9A2 gene. Am. J. Med. Genet. 77:234-240.

6. Paassilta, P., et al. 1999. COL9A3: a third locus for multiple epipyseal dysplasia. Am. J. Hum. Genet. 64:1036-1044.

7. Chapman, K.L., et al. 2001. Mutations in the region encoding the von Willebrand factor A domain of matrilin-3 are associated with multiple epiphyseal dysplasia. Nat. Genet. 28:393-396.

8. Czarny-Ratajczak, M., et. al. 2001. A mutation in COL9A1 causes multiple epiphyseal dysplasia: further evidence for locus heterogeneity. Am.J. Hum. Genet. 69:969-980.

9. Stanescu, V., Maroteaux, P., and Stanescu, R. 1982. The biochemical defect of pseudoachondroplasia. Eur. J. Ped. 138:221-225.

10. Maddox, B.K., et al. 1997. The fate of cartilage oligomeric matrix protein is determined by the cell type in the case of a novel mutation in pseudoachondroplasia. J. Biol. Chem. 272:30993-30997.

11. Hecht, J.T., et al. 1998. Characterization of cartilage oligomeric matrix protein (COMP) in human normal and pseudoachondroplasia musculoskeletal tissues. Matrix Biol. 17:269-278.

12. Maynard, J.A., Cooper, R.R., and Ponseti, I.V. 1972. A unique rough surfaced endoplasmic reticulum inclusion in pseudoachondroplasia. Lab. Invest. 26:40-44.

13. Delot, E., King, L.M., Briggs, M.D., Wilcox, W.R., and Cohn, D.H. 1999. Trinucleotide expansion mutations in the cartilage oligomeric matrix protein (COMP) gene. Hum. Mol. Genet. 8:123-128.

14. Mörgelin, M., Heinegård, D., Engel, J., and Paulsson, M. 1992. Electron microscopy of native cartilage oligomeric matrix protein purified from the Swarm rat chondrosarcoma reveals a five-armed structure. J. Biol. Chem. 267:6137-6141.

15. Zaia, J., et al. 1997. Posttranslational modifications in COMP. Characterization of the $\mathrm{N}$-linked oligosaccharides by matrix assisted laser desorption ionization in time-of-flight mass spectrometry. J. Biol. Chem. 272:14120-14126.

16. Müller, G., Michel, A., and Altenburg, E. 1998. COMP (cartilage oligomeric matrix protein) is synthesized in ligament, tendon, meniscus, and articular cartilage. Connect. Tissue Res. 39:233-244.

17. Newton, G., et al. 1994. Characterization of human and mouse cartilage oligomeric matrix protein. Genomics. 24:435-439.

18. Rosenberg, K., Olsson, H., Mörgelin, M., and Heinegård, D. 1998. Cartilage oligomeric matrix protein shows high affinity zinc-dependent interaction with triple helical collagen. J. Biol. Chem. 273:20397-20403.

19. Thur, J., et al. 2001. Mutations in cartilage oligomeric matrix protein (COMP) causing pseudoachondroplasia and multiple epiphyseal dysplasia affect binding of calcium and collagen I, II, and IX. J. Biol. Chem. 276:6083-6092.

20. Bleasel, J.F., et al. 1998. Changes in serum cartilage marker levels indicate altered cartilage metabolism in families with the osteoarthritis-related type II collagen gene COL2A1 mutation. Arthritis Rheum. 42:39-45.

21. Kühne, S.A., et al. 1998. Persistent high serum levels of cartilage oligomeric matrix protein in a subgroup of patients with traumatic knee injury. Rheumatol. Int. 18:21-25.

22. Månsson, B., et al. 1995. Cartilage and bone metabolism in rheumatoid arthritis. Differences between rapid and slow progression of disease 
identified by serum markers of cartilage metabolism. J. Clin. Invest. 95:1071-1077.

23. Svensson, L., et al. 2002. Cartilage oligomeric matrix protein-deficient mice have normal skeletal development. Mol. Cell. Biol. 22:4366-4371.

24. Hecht, J.T., et al. 1998. Retention of cartilage oligomeric matrix protein (COMP) and cell death in redifferentiated pseudoachondroplasia chondrocytes. Matrix Biol. 17:625-633.

25. Delot, E., Brodie, S.G., King, L.M., Wilcox, W.R., and Cohn, D.H. 1998 Physiological and pathological secretion of cartilage oligomeric matrix protein by cells in culture. J. Biol. Chem. 273:26692-26697.

26. Holden, P., et al. 2001. Cartilage oligomeric matrix protein interacts with type IX collagen, and disruptions to these interactions identify a pathogenetic mechanism in a bone dysplasia family. J. Biol. Chem. 276:6046-6055.

27. Chen, H., Deere, M., Hecht, J.T., and Lawler, J. 2000. Cartilage oligomeric matrix protein is a calcium-binding protein, and a mutation in its type 3 repeats causes conformational changes. J. Biol. Chem. 275:26538-26544

28. Maddox, B.K., Mokashi, A., Keene, D.R., and Bächinger, H.P. 2000. A cartilage oligomeric matrix protein mutation associated with pseudoachondroplasia changes the structural and functional properties of the type 3 domain. J. Biol. Chem. 275:11412-11417.

29. Dinser, R., et al. 2001. Comparison of long-term transgene expression after non-viral and adenoviral gene transfer into primary articular chondrocytes. Histochem. Cell. Biol. 116:69-77.

30.Zaucke, F., Dinser, R., Maurer, P., and Paulsson, M. 2001. Cartilage oligomeric matrix protein (COMP) and collagen IX are sensitive markers for the differentiation state of primary articular chondrocytes. Biochem. J. 358:17-24.

31. Kochanek, S. 1999. High-capacity adenoviral vectors for gene transfer and somatic gene therapy. Hum. Gene Ther. 10:2451-2459.

32. Häuselmann, H.J., et al. 1994. Phenotypic stability of bovine articular chondrocytes after long-term culture in alginate beads. J. Cell Sci. 107:17-27.

33. Klatt, A.R., et al. 2001. Molecular structure, processing, and tissue distribution of matrilin-4. J. Biol. Chem. 276:17267-17275.
34. Kreppel, F., Biermann, V., Kochanek, S., and Schiedner, G. 2002. A DNAbased method to assay total and infectious particle contents and helper virus contamination in high-capacity adenoviral vector preparations. Hum. Gene Ther. 13:1151-1156.

35. Mosmann, T. 1983. Rapid colorimetric assay for cellular growth and survival: application to proliferation and cytotoxicity assays. J. Immunol. Methods. 65:55-63.

36. Wada, I., Kai, M., Imai, S., Sakane, F., and Kanoh, H. 1997. Promotion of transferrin folding by cyclic interactions with calnexin and calreticulin. EMBO J. 16:5420-5432.

37. Laquerre, S., Anderson, D.B., Argnani, R., and Glorioso, J.C. 1998. Herpes simplex virus type 1 glycoprotein $B$ requires a cysteine residue at position 633 for folding, processing, and incorporation into mature infectious virus particles. J. Virol. 72:4940-4949.

38. Hultenby, K., Reinholt, F.P., and Heinegård, D. 1993. Distribution of integrin subunits on rat metaphyseal osteoclasts and osteoblasts. Eur.J. Cell Biol. 62:86-93.

39. Lehrman, M.A. 2001. Oligosaccharide-based information in endoplasmic reticulum quality control and other biological systems. J. Biol. Chem. 276:8623-8626.

40. Kim, P.S. and Arvan, P. 1998. Endocrinopathies in the family of endoplasmic reticulum (ER) storage diseases: disorders of protein trafficking and the role of ER molecular chaperones. Endocrine Rev. 19:173-202.

41. Ekman, S., Reinholt, F.P., Hultenby, K., and Heinegård, D. 1997. Ultrastructural immunolocalization of cartilage oligomeric matrix protein (COMP) in porcine growth cartilage. Calcif. Tissue Int. 60:547-553.

42. Murphy, J.M., Heinegård, D., McIntosh, A., Sterchi, D., and Barry, F.P. 1999. Distribution of cartilage molecules in the developing mouse joint. Matrix Biol. 18:487-497.

43. Kuznetsov, G., Chen, L.B., and Nigam, S.K. 1997. Multiple molecular chaperones complex with misfolded large oligomeric glycoproteins in the endoplasmic reticulum. J. Biol. Chem. 272:3057-3063.

44. Rosenberg, K. 2001. Cartilage oligomeric matrix protein (COMP): functions in collagen binding and assembly. Ph.D. thesis. Lund University. Lund, Sweden. 64 pp. 